\title{
PREDICTION OF COGNITIVE STATUS AND 5-YEAR SURVIVAL RATE FOR ELDERLY WITH CARDIOVASCULAR DISEASES: A CANADIAN STUDY OF HEALTH AND AGING SECONDARY DATA ANALYSIS
}

\author{
S. PAKZAD ${ }^{1}$, P. BOURQUE ${ }^{1}$, N. FALLAH ${ }^{2}$ \\ 1. École de psychologie, Université de Moncton, 18 Antonine-Maillet Avenue, Moncton, New Brunswick, Canada E1A 3E9; 2. Division of Neurology, Department of Medicine, \\ University of British Columbia, UBC Hospital, Vancouver, British Columbia, Canada, V6T 2B5 \\ Corresponding author: Dr. Sarah Pakzad, Ph.D. 531, Taillon, Université de Moncton, Moncton, NB E1A 3E9, Tel: (506) 858-4245; Fax: (506) 852-3125, \\ Email: sarah.pakzad@umoncton.ca
}

\begin{abstract}
Background: Given the important association between cardiovascular disease and cognitive decline, and their significant implications on frailty status, the contribution of neurocognitive frailty measure helping with the assessment of patient outcomes is dearly needed. Objectives: The present study examines the prognostic value of the Neurocognitive Frailty Index (NFI) in the elderly with cardiovascular disease. Design: Secondary analysis of the Canadian Study of Health and Aging (CSHA) dataset was used for prediction of 5-year cognitive changes. Setting: Community and institutional sample. Participants: Canadians aged 65 and over [Mean age: 80.4 years (SD=6.9; Range of 66-100)]. Measurement: Neurocognitive Frailty Index (NFI) and Modified Mini-Mental State (3MS) scores for cognitive functioning of all subjects at follow-up and mortality rate were measured. Results: The NFI mean score was $9.63(\mathrm{SD}=6.04)$ and ranged from 0 to 33. This study demonstrated that the NFI was significantly associated with cognitive changes for subjects with heart disease and this correlation was a stronger predictor than age. Conclusion: The clinical relevance of this study is that our result supports the prognostic utility of the NFI tool in treatment planning for those with modifiable cardiovascular disease risk factors in the development of dementia.
\end{abstract}

Key words: Frailty, cardiovascular disease, mortality, cognitive deficits.

\section{Introduction}

While cardiovascular diseases (CVD) are associated to cognitive decline and dementia in the elderly $(1,2)$, much consideration has been given to the contribution of cardiovascular diseases to cognitive impairment (3). Today, CVD are established to strongly relate to cognitive impairment and dementia in the elderly (1). Interestingly, CVD can lead to Alzheimer's disease (AD) and vascular dementia (1).

Coronary heart disease, hypertension, left ventricular hypertrophy, among others, are risk factors for heart failure (4). The association between CVD and cognitive impairment in older people is multifactorial and involves common risk factors such as atherosclerosis, hypertension, and diabetes mellitus (1). A large European longitudinal study (5) has shown that modifiable cardiovascular risk factors (CRFs) including diabetes, smoking, hypertension, and low physical activities are associated with lower cognitive test scores. A review (3) additionally underscored that traditional CRFs (e.g., high blood pressure, hyperglycemia, insulin resistance, diabetes mellitus, hyperlipidemia, cigarette smoking, heart failure, stroke, and atrial fibrillation) are also risk factors for AD. However, it is unclear whether those modifiable behaviors significantly partake in lowering the risk of developing cognitive impairment (6). As highlighted, under promising strategies for prevention of dementia (7), these risk factors need to be identified in vulnerable individuals so that appropriate preventative interventions can be undertaken to reduce the risk of developing cognitive impairment. Indeed, evidence shows that healthy cardiovascular lifestyles mitigate cognitive disorders through modifiable CVD risk factors (8).

Growing evidence shows that vascular/cerebrovascular pathology can accelerate the progression of preclinical $\mathrm{AD}$ and speed disease evolution (9). For instance, one study (10) note that those who have CVD are at a greater risk of developing mild cognitive impairment (MCI), and another review and synthesis, examining the pathophysiologic relationship between $\mathrm{AD}$, cerebrovascular disease, and cardiovascular risk [9] showed that effectively controlling vascular risk factors serves to delay onset of $\mathrm{AD}$ and that the management of CRFs is associated with a reduced risk of dementia.

Frailty, an age-related fragility associated with multidimensional loss of energy, physical ability, cognition and general health reserves (11), has been positively associated with cognitive function in individuals with CVD (12) and is more prevalent in patients with CVD (13). To this end, it is noteworthy of underscoring the significance of the bidirectional relationship between frailty and both cognitive and functional reserve, and implication in neuropathology and brain reserve, motor signs of aging and the reversibility of cognitive frailty $(14,15)$. Hence, we need to underscore the importance of a frailty index as a prognostic tool to evaluate the risk of cognitive impairment and death.

Among those high-risk groups, individuals with 


\section{PROGNOSIS OF COGNITIVE STATUS AND SURVIVAL RATE USING CSHA DATA}

cardiovascular health conditions are more vulnerable to the development of dementia (16). Thus, index of 'frailty' needs to be considered in cognitive decline as a modifying and prognostic variable since morbidity and mortality are related to CVD (17). The association between CVD and cognitive impairment have long been established using statistical approach whereas multidimensional approach is lagging behind that could confirm this association.

Recently, the Neurocognitive Frailty Index (NFI) was developed to create a more comprehensive measure of frailty in the elderly (11), and evidence supports its higher accuracy compared to other frailty outcome measures (11). Although evidence shows that the NFI provides higher accuracy to predict outcomes, the contribution of neurocognitive frailty indicator in improving patient prognostic in high-risk groups has not been established, that subsequently warrants further studies (11).

Given the link between CVD and dementia, there is an urgent need to examine these associations (18). Thus, a scientific group of experts convened by the Alzheimer's Association, with scientific input from the National Institute of Neurological Disorders and Stroke and the National Heart, Lung and Blood Institute from the National Institutes of Health (2) to help in developing more accurate outcome measures and clinical criteria to examine the effects of controllable vascular risk factors for developing AD. Furthermore, the risk of developing MCI is higher for people who have CVD (19) or CRFs (20). This knowledge is important because this can help in examining ways to prevent or better manage the precursors of dementia. As noted earlier (11), studies to further evaluate the contribution of neurocognitive frailty indicators in improving prediction of patient outcomes are needed. Hence, this study evaluates the prognostic value of the NFI in the elderly with cardiovascular disease.

\section{Materials and Methods}

\section{Study population}

Participants' data were drawn from the Canadian Study of Health and Aging (CSHA) (21), a study of the epidemiology of dementia in Canada. Planned in 1989, as a national longitudinal study, the CSHA followed over 10,000 elderly Canadians (from 36 communities and institutions) over a ten-year period from 1991 to 2001 and has collected a wide range of information on changing health status over time [http://www.csha.ca]. The CSHA was conducted in three waves but in this study only data from waves 1 and 2 were used: CSHA-1 (1991 to 1992) and CSHA-2 (1996 to 1997) for prediction of 5-year cognitive changes. The current analysis focused on the 997 participants who received a consensus diagnosis of no-cognitive impairment (NCI) or cognitive impairment but not dementia (CIND) on CSHA-1. Samples included those who completed neuropsychological tests at CSHA-1 and received a clinical diagnostic assessment at CSHA-1 and CSHA-2 $(n=1228)$. The CIND category included individuals whose level of cognitive impairment was measured to be greater than the NCI group but less than the dementia group. Of the original 997 participants, 299 individuals had died in five years of follow-up, but these were included in our participant sample.

\section{Measurement}

NFI was defined as a combined score of 42 physical and cognitive elements. The physical elements included 4 domains, activities of daily living, instrumental activities of daily living, general health and comorbidities. The cognitive elements include 8 domains, short-term memory, long-term memory, verbal abstract thinking, judgment, aphasia, apraxia, agnosia, and constructional difficulty, as they were available in the dataset. Global cognitive score was obtained via the 3MS (22) at baseline and at follow-up, and mortality rate was used as an outcome measure.

\section{Coding of Neurocognitive Frailty Index}

The initial NFI (11) was determined from 42 variables, here we used 41 variables, as CVD was our chief focus. The NFI variables, which include both physical and cognitive elements, are described in our earlier study (11). Thirty-three physical components were selected from the CSHA-1 dataset at baseline (1991). Binary variables were recoded, using the " 0 ", to indicate the absence of the deficit, and " 1 " to indicate the presence of a deficit. Particularly in Activities of Daily Living (ADL) and Instrumental Activities of Daily Living (IADL) items, data converted as score "1" for "can't do at all" or "with some help" denoting needed help, and we assigned score "0" for "without any help". The Self-Rated Health Question, «How is your health these days? Very good, pretty good, not too good, poor, very poor» was rated between " 0 " and " 1 ". Each lower self-rating of health was coded to represent a larger deficit «very good $=0 »$, «pretty good $=0.25 »,\langle$ good $=0.5 »$, «poor $=0.75 »$ and «very poor=1». For variables with dichotomous response (general health), data coded into a score between " 0 " where no deficit is present and " 1 " where the deficit is present. Overall, physical component scores varied between zero " 0 " to thirtythree " 33 ".

Similarly, we recoded the cognitive component but by recognizing an ordinal scale of variables. A simple recoding was done for the eight elements: short-term memory, long-term memory, verbal abstract thinking, judgment, aphasia, apraxia, agnosia, and constructional difficulty. A score was assigned a "0" for response "None", " 1 " for "Questionable", "2" for "Mild", " 3 " for "Moderate" and " 4 " for "Severe". There were eight measures in this part which means theoretically that a person could have a score between zero " 0 " to thirty-two " 32 ". Each of the 41 components added together, therefore there was a total score of NFI ranging between zero " 0 " and sixty-five "66". The physical and cognitive variables description and cut points for the NFI are presented in a previous article (11). 
THE JOURNAL OF FRAILTY \& AGING

Table 1

Demographic characteristics and cognitive status of the sample as a function of NFI score categories

\begin{tabular}{|c|c|c|c|c|c|}
\hline NFI score * & $0-3$ & 4-9 & $10-17$ & $>=18$ & P-value \\
\hline Male $(\%)$ & $99(50.8)$ & 191 (47.6) & $100(33)$ & $25(25.5)$ & 0.001 \\
\hline 3MS-CSHA1 & $89(8.3)$ & $84.9(9.6)$ & $79(9.5)$ & $72.1(9.6)$ & 0.001 \\
\hline 3MS-CSHA2 & $86.7(12.6)$ & $78.9(14.5)$ & $68.9(18.2)$ & $58.1(22.2)$ & 0.001 \\
\hline
\end{tabular}

Note: 3MS: Modified Mini-Mental State; CSHA: Canadian Study of Health and Aging; NFI: Neurocognitive Frailty Index. Data showing mean (standard deviation) for all variables, and total number (percentage) for sex category. * NFI score defined as number of deficits for example " $0-3$ " means zero to three deficits

Table 2

Associations between NFI and Cognitive status (3MS at follow-up) using Multiple Linear regression for participants without CVD in Panel A and with CVD in Panel B

Panel A

\begin{tabular}{lcccc}
\hline Variables & B $^{*}$ & SE* $^{*}$ & Standardized B & P- value \\
\hline Age & -0.596 & 0.099 & -0.218 & 0.001 \\
Male (\%) & -3.114 & 1.277 & -0.085 & 0.015 \\
Education (years) & 0.371 & 0.177 & 0.084 & 0.037 \\
3MS (baseline) & 0.863 & 0.078 & 0.484 & 0.001 \\
NFI (continuous) & -0.600 & 0.142 & -0.170 & 0.001 \\
\hline
\end{tabular}

*Note: B: Beta coefficient; SE: Standard Error.

Panel B

\begin{tabular}{lcccc}
\hline Variables & B $^{*}$ & SE $^{*}$ & Standardized B & P-value \\
\hline Age & -0.519 & 0.134 & -0.208 & 0.001 \\
Male (\%) & -3.114 & 1.277 & -0.085 & 0.359 \\
Education (years) & 0.579 & 0.266 & 0.135 & 0.001 \\
3MS (baseline) & 0.788 & 0.114 & 0.473 & 0.001 \\
NFI (continuous) & -0.797 & 0.188 & -0.250 & 0.001 \\
\hline
\end{tabular}

*Note: B: Beta coefficient; SE: standard Error.

\section{Statistical Analysis}

The parametric tests were used for normally distributed variables and nonparametric tests were used for the not normally distributed. Before applying regression models univariate tests (including chi-square, t-test, ANOVA, correlation) were used for data exploration.

Categorical NFI scores were created using classification and regression trees, using the applied Chi-squared Automatic Interaction Detector (CHAID) model. This modeling accounts for the binomial distributions in response variable. Validation of the multivariate analyses was tested with a 10-fold crossvalidation; CHAID identifies 'nodes', or persons subgroups, that are most homogeneous with regards to probability of death. These nodes were then applied to Cox regression for further investigation. In addition to bivariate tests and decision tree, two separate Cox regression models were applied to the data to estimate chance of death in 5 years of follow-up. Cox regression was used in two formats of NFI (one for NFI as a continuous and another as a categorical variable). A p-value of 0.05 or lower was considered statistically significant. Statistical analyzes were performed using SPSS (version 23) (23) and $\mathrm{R} \times 64$ (version 3.1) (24).

\section{Results}

This was a secondary analysis of the Canadian Study of Health and Aging (CSHA) dataset $(21,25)$. The NFI mean was $9.63(\mathrm{SD}=6.04)$ and ranged from 0 to 33 . Distribution of NFI 


\section{PROGNOSIS OF COGNITIVE STATUS AND SURVIVAL RATE USING CSHA DATA}

was slightly skewed to the right (skewness $=0.95, \mathrm{SE}=0.08$; Kurtosis $=0.90, \mathrm{SE}=0.16$ ). At baseline, the mean age of sample was 80.4 years (SD=6.9; Range: 66-100). There was a clear positive, yet small association between age and NFI at baseline (rho=0.27, $\mathrm{p}<0.001,2$-tailed).

Presented in Table 1, demographic characteristics and cognitive data were described using means and standard deviation for age, 3MS at baseline (CSHA1) and at followup (CSHA2), years of education, and male sex percentage, as a function of NFI scores breakdown. Here, NFI result was grouped into 4 categories based on decision trees results (Figure 1).

Tables 2 presents the results of two separate regression models predicting outcomes $3 \mathrm{MS}$ for participants with and without CVD. In both models, NFI is significantly related to 3MS at follow-up; however, NFI is stronger predictor in people with CVD than without. In the multiple linear regression analysis, adjusted for age, sex, education, and $3 \mathrm{MS}$ at baseline, the NFI was correlated to $3 \mathrm{MS}$ at followup (regression coefficient $=-0.6)$, and age $(\mathrm{p}<0.05)$. Every additional deficit used to calculate the NFI was associated with an increased chance of cognitive decline. However, this association was stronger in people with CVD. NFI (regression coefficient $=-0.79$ ) was significantly associated with cognitive status at follow-up for people with CVD and this was stronger than age $(\mathrm{p}<0.05)$.

Although in both groups (with and without heart problems), $\mathrm{NFI}$ is related to the outcome (without: $\mathrm{R} 2=0.08$, rho $=0.32$, $\mathrm{p}<0.01$; with:R2 $=0.02$, rho $=0.141, \mathrm{p}<0.05)$, the association between NFI and $3 \mathrm{MS}$ indicates different variability to predict the outcome in people with and without CVD (Table 2).

The CHAID decision tree analysis was used to determine optimal stratification groups to best identify association between NFI and mortality. Result indicated that the NFI could be grouped into four categories. Group one (category) is for the people who have an NFI score of less than 2.75 deficits and mortality rate of $7.6 \%$. The second and third groups have a mortality rate of $21.8 \%$ and $36.3 \%$, respectively, indicating a three to five times more mortality rate, compared to baseline. The highest risk was for people with an NFI score of more than 17.8 and mortality rate of $58 \%$. We used this result for making 4 levels of NFI to predict mortality.

Cox regression was applied for modeling probability of mortality when NFI was considered as a continuous variable. NFI was significantly related to mortality probability with an odds ratio of 1.08 . This indicates participants with a higher NFI score have a higher mortality probability. In this model, age, gender and CVD were significant (Table 3, Panel A). In the proportional hazard ratio analysis, accounting for age, gender and education as confounding variables, the value of NFI (as categorical) was more highly correlated to survival than age. Every additional deficit used to calculate the NFI was associated with an increased risk. By using results of the decision tree grouping, the NFI was significantly associated with mortality for the second group of participants in comparison to the fittest people at baseline with an NFI score between 4 and 8 deficits (HR $=2.63,95 \%$ confidence interval (CI) $1.22-5.71 ;$ p-value $=0.014)$. Participants with $18+$ NFI deficits showed greater risk $[\mathrm{HR}=9.172,(95 \% \mathrm{CI} 4.12-20.41)]$, indicating they have 9 times more chance of death compared

Figure 1

Decision trees to determine optimal stratification of NFI for predicting mortality

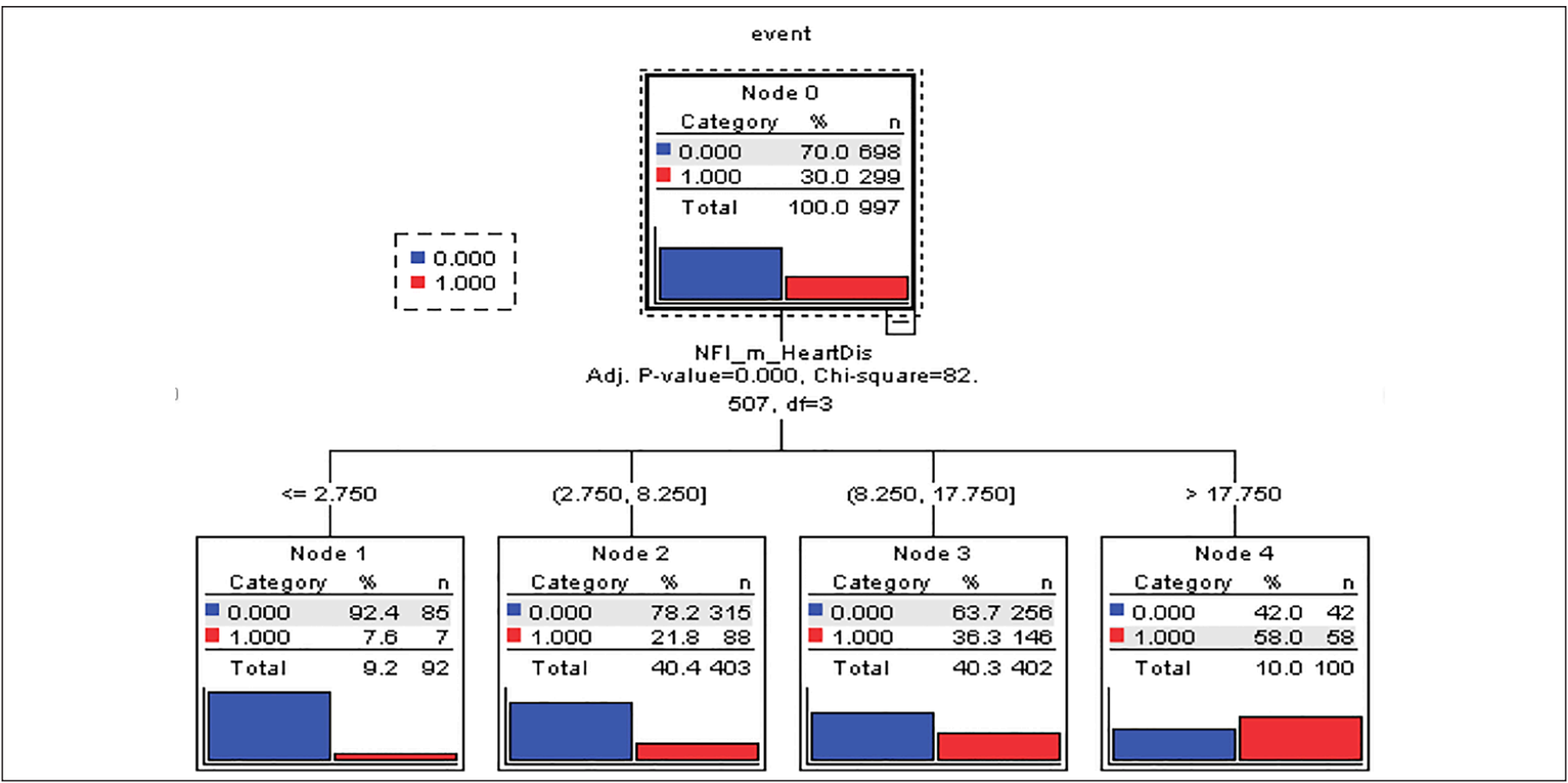


Table 3

Association between NFI and mortality probability using Cox proportional hazard model (Panel A). Association between NFI and mortality using Cox proportional hazard model (Panel B)

Panel A

\begin{tabular}{lccccc}
\hline Variables & B $^{*}$ & SE* $^{*}$ & HR* $^{*}$ & $\mathbf{9 5 \%} \mathbf{C I}^{*}$ & P value \\
\hline Age & 0.049 & 0.009 & 1.050 & $1.032-1.068$ & 0.001 \\
Male $(\%)$ & 0.539 & 0.119 & 1.714 & $1.357-2.163$ & 0.001 \\
CVD & 0.269 & 0.121 & 1.308 & $1.033-1.658$ & 0.026 \\
NFI (continuous) & 0.073 & 0.009 & 1.076 & $1.057-1.094$ & 0.001 \\
\hline
\end{tabular}

*Note: B: Beta coefficient; CI: Confidence Interval; HR: Hazard Ratio; SE: standard Error.

Panel B

\begin{tabular}{lccccc}
\hline Variables & B $^{*}$ & SE* $^{*}$ & HR* & 95\% CI* & P value \\
\hline Age & 0.048 & 0.009 & 1.049 & $1.031-1.049$ & 0.001 \\
Male $(\%)$ & 0.563 & 0.120 & 1.757 & $1.389-2.222$ & 0.001 \\
CVD & 0.250 & 0.122 & 1.284 & $1.012-1.630$ & 0.040 \\
NFI ( $\leq 3$ deficits)* & ------- & ------ & ------ & ------------ & ------ \\
NFI (4-8 deficit) & 0.970 & 0.394 & 2.638 & $1.218-5.714$ & 0.014 \\
NFI (9-17 deficit) & 1.541 & 0.391 & 4.669 & $2.169-10.051$ & 0.001 \\
NFI ( $\geq 18$ deficits) & 2.216 & 0.408 & 9.172 & $4.122-20.409$ & 0.001 \\
\hline
\end{tabular}

*Note: B: Beta coefficient; CI: Confidence Interval; HR: Hazard Ratio; NFI ( $\leq 3$ deficits) is baseline. SE: standard Error.

to baseline (Table 3, Panel B). Moreover, mortality probability was greater in people with CVD $(H R=1.28)$ than without (Figure 2, Panel A). Although all levels of NFI were highly correlated to mortality (Figure 2, Panel B), the Kaplan-Meier curve shows increasing level of NFI, suggesting dose-response effect in relation to survival.

\section{Discussion}

Our study aimed to predict cognitive status and 5-year survival rate in participants with CVD in the Canadian Study of Health and Aging (21) dataset using a deficit accumulation approach. The NFI, as a measure of frailty, was used to examine the association between frailty and cognition as measured by $3 \mathrm{MS}$ at baseline and 5 years later in individuals with and without CVD.

We have shown a significant and positive association between age and NFI, indicating that neurocognitive frailty increases with age. Additionally, the magnitude of the association for the NFI was larger than age. Furthermore, higher NFI score was associated with higher probability of mortality. Mortality was $28 \%$ greater in individuals with than without CVD.

Our study results support previous findings from various scientific groups. For example, the Alzheimer's Association that noted CVD are present in most cases with $\mathrm{AD}$, without suggesting that all individuals with $\mathrm{AD}$ have heart problems (9). Similarly, the Cardiovascular Health Study Cognition
Study $(26,27)$ found subjects with mild cognitive deficits have CVD. Using the results of models from this study, baseline cognition and age were significant predictors, yet a more comprehensive prediction can be achieved by including frailty measures. Additionally, our results concur with studies examining vascular risk factors involving cognitive decline. A longitudinal study (28) found the associations between smoking and long-term blood pressure with the risk of cognitive decline. However, a review (3) underlined the need for more sensitive neuropsychological measures. Our study shows that the NFI serves as a more effective prognostic tool to evaluate the risk of developing dementia. Findings from an earlier study (29) underscore the association between vascular risk factors and cognitive function at midlife, and their different impacting pathways on brain mechanisms. Although we have shown that the NFI was highly correlated to mortality, a dose-response effect in relation to survival is noted with increasing levels of NFI. Thus, the identification of CRFs using the NFI might serve to identify those at higher risk for dementia so to provide preventative interventions.

Perhaps the most important implication of our result is the integration of physical frailty and cognitive elements as a neurocognitive frailty measure such as the NFI to better understand the relationship between CVD and cognitive decline. Indeed, in line with our findings, others (12) have noted the importance of including the frailty status in the clinical process of evaluating risk factors for dementia to optimize the treatment plan. The present study provides validation-evidence 


\section{PROGNOSIS OF COGNITIVE STATUS AND SURVIVAL RATE USING CSHA DATA}

for the use of NFI with elderly individuals at risk for cognitive deficit with co-existing CVD to enhance the prognostic value of cardiovascular risk factors.

\section{Figure 2}

Kaplan-Meier curves for the proportional survival of people with and without CVD (Panel A) also with various levels of NFI (Panel B). In Panel B, node 1 indicate NFI $<4$ and node 2 for NFI between 4 and 8 , node 3 related to NFI score between 9 and 17 , and finally node 4 is related to NFI more than 18 score

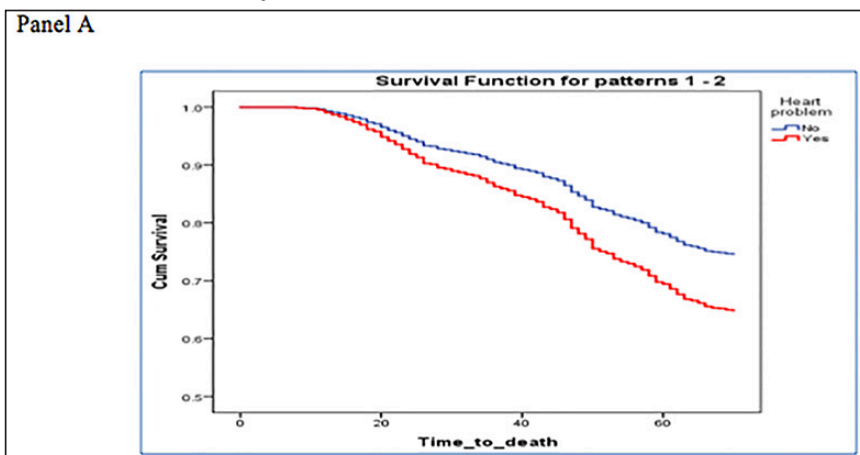

Panel B

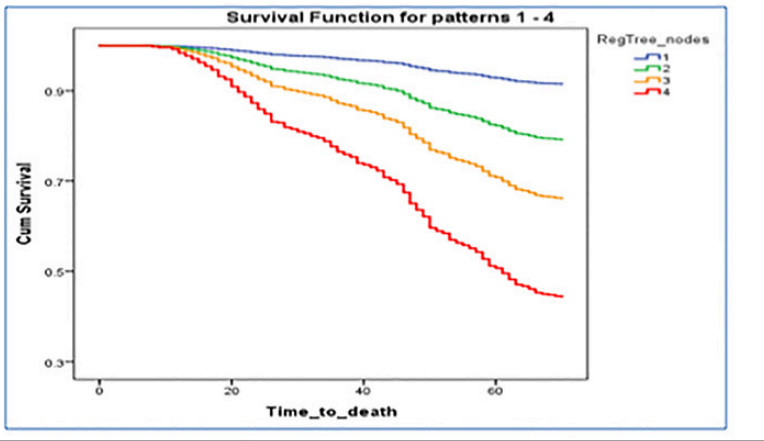

Noteworthy that our study is not without limitation, and we can underscore our use of cognitive screening tool as a possible issue. In our study the cognitive status of all subjects was assessed through the Modified Mini-Mental State test; although this test offers increased validity over the MMSE, it remains a screening tool for the evaluation of cognitive impairment.

We have established an association between CVD and cognitive deficits; however, further investigation is needed to clarify the extent and types of modifiable CVD risk factors (e.g., diabetes and hypertension) and their consequence on the cognitive functions. As previously highlighted (30) to identify individuals needing specific intervention plans and to maximize outcomes based on risk profiles using the NFI future research is warranted. Since the NFI risk profile could be useful in treatment planning, and that CVD are related to cognitive deficits as noted by several colleagues (31), maintaining vessel elasticity through physical exercise could be beneficial to those at risk of developing cognitive deficits.

Conflict of Interest: None to declare.
Sponsors' role: None to declare

Disclosure: The research team would like to acknowledge the access given to the Canadian Study of Health and Aging (CSHA) dataset by Dr Kenneth Rockwood, which was much appreciated.

Ethical Standards: The authors declare that the experiments and data collected comply with the current laws of the country in which they were performed.

Open Access: This article is distributed under the terms of the Creative Commons Attribution 4.0 International License (http://creativecommons.org/licenses/by/4.0/), which permits use, duplication, adaptation, distribution and reproduction in any medium or format, as long as you give appropriate credit to the original author(s) and the source, provide a link to the Creative Commons license and indicate if changes were made.

\section{References}

1. Abete, P., D. Della-Morte, G. Gargiulo, et al., Cognitive impairment and cardiovascular diseases in the elderly. A heart-brain continuum hypothesis. Ageing Res Rev, 2014. 18: p. 41-52.

2. Snyder, H.M., R.A. Corriveau, S. Craft, et al., Vascular contributions to cognitive impairment and dementia including Alzheimer's disease. Alzheimers Dement, 2015. 11(6): p. 710-7.

3. Gorelick, P.B., S.E. Counts, and D. Nyenhuis, Vascular cognitive impairment and dementia. Biochim Biophys Acta, 2016. 1862(5): p. 860-8.

4. Bressler, J., D.S. Knopman, A.R. Sharrett, et al., Incident heart failure and cognitive decline: The atherosclerosis risk in communities study. J Card Fail, 2017. 23(1): p. $47-55$

5. Arntzen, K.A., H. Schirmer, T. Wilsgaard, and E.B. Mathiesen, Impact of cardiovascular risk factors on cognitive function: the Tromso study. Eur J Neurol, 2011. 18(5): p. 737-43.

6. Yaffe, K., Metabolic syndrome and cognitive disorders: is the sum greater than its parts? Alzheimer Dis Assoc Disord, 2007. 21(2): p. 167-71

7. Middleton, L.E. and K. Yaffe, Promising strategies for the prevention of dementia. Arch Neurol, 2009. 66(10): p. 1210-5.

8. Gonzalez, H.M., W. Tarraf, K. Harrison, et al., Midlife cardiovascular health and 20-year cognitive decline: Atherosclerosis Risk in Communities Study results. Alzheimers Dement, 2018. 14(5): p. 579-589.

9. Santos, C.Y., P.J. Snyder, W.C. Wu, et al., Pathophysiologic relationship between Alzheimer's disease, cerebrovascular disease, and cardiovascular risk: A review and synthesis. Alzheimers Dement (Amst), 2017. 7: p. 69-87.

10. McLennan, S.N., J.L. Mathias, L.C. Brennan, and S. Stewart, Validity of the Montreal Cognitive Assessment (MoCA) as a screening test for mild cognitive impairment (MCI) in a cardiovascular population. J Geriatr Psychiatry Neurol, 2011. 24(1): p. 33-8.

11. Pakzad, S., P. Bourque, H. Feldman, and N. Fallah, Toward developing a novel Neurocognitive Frailty Index in the elderly. Journal of Geriatric Medicine and Gerontology, 2017.3(3): p. 032.

12. Weinstein, G., M. Lutski, U. Goldbourt, and D. Tanne, Physical frailty and cognitive function among men with cardiovascular disease. Arch Gerontol Geriatr, 2018. 78: p. $1-6$.

13. von Haehling, S., S.D. Anker, W. Doehner, J.E. Morley, and B. Vellas, Frailty and heart disease. Int J Cardiol, 2013. 168(3): p. 1745-7.

14. Facal, D., A. Maseda, A.X. Pereiro, et al., Cognitive frailty: A conceptual systematic review and an operational proposal for future research. Maturitas, 2019. 121: p. 48-56.

15. Dedeyne, L., M. Deschodt, S. Verschueren, J. Tournoy, and E. Gielen, Effects of multi-domain interventions in (pre)frail elderly on frailty, functional, and cognitive status: a systematic review. Clin Interv Aging, 2017. 12: p. 873-896.

16. de la Torre, J.C., Cardiovascular risk factors promote brain hypoperfusion leading to cognitive decline and dementia. Cardiovasc Psychiatry Neurol, 2012. 2012: p. 367516.

17. Singh, M., R. Stewart, and H. White, Importance of frailty in patients with cardiovascular disease. Eur Heart J, 2014. 35(26): p. 1726-31.

18. Suemoto, C.K., R.E. Ferretti, L.T. Grinberg, et al., Association between cardiovascular disease and dementia. Dement Neuropsychol, 2009. 3(4): p. 308-314

19. Barnes, D.E., G.S. Alexopoulos, O.L. Lopez, J.D. Williamson, and K. Yaffe, Depressive symptoms, vascular disease, and mild cognitive impairment: findings from the Cardiovascular Health Study. Arch Gen Psychiatry, 2006. 63(3): p. 273-9.

20. Kivipelto, M., E.L. Helkala, T. Hanninen, et al., Midlife vascular risk factors and latelife mild cognitive impairment: A population-based study. Neurology, 2001. 56(12): p. 1683-9.

21. Canadian Study of Health and Aging: Study methods and prevalence of dementia. CMAJ, 1994. 150(6): p. 899-913. 


\section{THE JOURNAL OF FRAILTY \& AGING}

22. Teng, E.L and H.C. Chui, The Modified Mini-Mental State (3MS) examination. J Clin Psychiatry, 1987. 48(8): p. 314-8.

23. IBM Corp, IBM SPSS Statistics for Windows. 2015, IBM Corp.: Armonk, NT.

24. R Development Core Team, R: a language and environment for statistical computing. 2014, R Foundation for Statistical Computing: Vienna, Austria.

25. The incidence of dementia in Canada. The Canadian Study of Health and Aging Working Group. Neurology, 2000. 55(1): p. 66-73.

26. Lopez, O.L., W.J. Jagust, C. Dulberg, et al., Risk factors for mild cognitive impairment in the Cardiovascular Health Study Cognition Study: part 2. Arch Neurol, 2003. 60(10): p. 1394-9.

27. Lopez, O.L., W.J. Jagust, S.T. DeKosky, et al., Prevalence and classification of mild cognitive impairment in the Cardiovascular Health Study Cognition Study: part 1. Arch Neurol, 2003. 60(10): p. 1385-9.

28. Dregan, A., R. Stewart, and M.C. Gulliford, Cardiovascular risk factors and cognitive decline in adults aged 50 and over: a population-based cohort study. Age Ageing, 2013. 42(3): p. 338-45.
29. Knopman, D.S., R.F. Gottesman, A.R. Sharrett, et al., Midlife vascular risk factors and midlife cognitive status in relation to prevalence of mild cognitive impairment and dementia in later life: The Atherosclerosis Risk in Communities Study. Alzheimers Dement, 2018. 14(11): p. 1406-1415.

30. Rosenberg, A., T. Ngandu, M. Rusanen, et al., Multidomain lifestyle intervention benefits a large elderly population at risk for cognitive decline and dementia regardless of baseline characteristics: The FINGER trial. Alzheimers Dement, 2018. 14(3): p. 263-270.

31. Gauthier, C.J., M. Lefort, S. Mekary, et al., Hearts and minds: Linking vascular rigidity and aerobic fitness with cognitive aging. Neurobiol Aging, 2015. 36(1): p. 304-14. 